\title{
Incidence and risk factors for symptoms from the eyes among professional computer users
}

\author{
Toomingas $\mathrm{A}^{\mathrm{a}, \mathrm{b}}{ }^{1}$, Hagberg $\mathrm{M}^{\mathrm{c}}$, Heiden $\mathrm{M}^{\mathrm{b}}$, Richter $\mathrm{H}^{\mathrm{b}}$, Westergren $\mathrm{KE}^{\mathrm{b}}$ and Wigaeus Tornqvist $\mathrm{E}^{\mathrm{b}, \mathrm{d}}$ \\ a Institute of Environmental Medicine, Karolinska Institutet, Sweden \\ $b$ Centre for Musculoskeletal Research, University of Gävle, Sweden \\ c Occupational \& Environmental Medicine University of Gothenburg, Sweden \\ $d$ School of health Sciences, Jönköping University, Sweden
}

\begin{abstract}
Personal computers are used by a majority of the working population in their professions. Little is known about risk-factors for incident symptoms from the eyes among professional computer users. The aim was to study the incidence and risk-factors for symptoms from the eyes among professional computer users.

This study is a part of a comprehensive prospective follow-up study of factors associated with the incidence of symptoms among professional computer users. 1531 computer users of different professions at 46 companies were invited, whereof 1283 answered a baseline questionnaire (498 men; 785 women) and 1246 at least one of 10 monthly follow-up questionnaires. The computer work-station and equipment were generally of a good standard. The majority used CRT displays.

During the follow-up period 329 subjects reported eye symptoms. The overall incidence rate in the whole study group was 0.38 per person-year, 0.23 in the subgroup of subjects who were symptom free at baseline and 1.06 among subjects who reported eye symptoms at baseline. In the bivariate analyses significant associations were found with all explanatory variables, except BMI. The reduced multivariate model showed significant associations with extended computer work, visual discomfort (dose-response), eye symptoms at baseline (higher risk), sex (women=higher risk) and nicotine use.

The incidence of eye problems among professional computer users is high and related to both individual and work-related factors.
\end{abstract}

Keywords: office work, eye strain, prospective study

\section{Introduction}

Personal computers are used by large groups of the working population in their professions, by students and in private life. Different health-related problems have been studied and reported, e.g. musculoskeletal disorders. Problems connected to the eyes are also reported. Most studies of eye-related disorders are cross-sectional, however. Little is known about riskfactors for incident symptoms from the eyes among professional computer users.

\section{Aim}

The aim was to study the incidence and riskfactors for symptoms from the eyes among professional computer users.

\section{Methods}

This study is a part of a comprehensive prospective follow-up study of factors associated with incidence of disorders among professional computer users in Sweden at the end of the 1990-ies. Overall descriptions of the study have been reported elsewhere $[1,2] .1531$ computer users of different professions

\footnotetext{
${ }^{1}$ Corresponding author. E-mail: allan.toomingas@ki.se
} 
(medical secretaries, receptionists, administrators, civil engineers, graphical editors, call centre operators etc) at 46 companies were enrolled, whereof 1283 answered the baseline questionnaire (498 men and 785 women) constituting the stud population, whereof 1246 participated in at least one follow-up (97\%). The working environments and equipments varied but were generally of good a standard of design and quality. The majority used CRT displays.

The baseline questionnaire asked about working conditions and individual factors. Age, sex, body height and weight (calculated as BMI), nicotine use and educational level were asked about. Mental demands and authority over decisions according to the Demand-Control model were rated on scales with 4 categories (Yes, often up to No, almost never). Two index were calculated: Demands (5 items) and Control (6 items). Subjects were also asked about the number of working hours and how many percent of these were spent doing computer work (recalculated to hours/day - ComputerTime; 3 levels) and how many percent of the computer-time they were using an input device - mouse, trackball etc. (recalculated to hours/day - DeviceTime; 3 levels). Subjects were also asked for how long time they do computer work without having a minimum 10 minutes break ( 7 categories: $<1$ hour up to $>6$ hours) and how often this happens (4 categories: rarely up to daily). A combined variable (ContinousTime) was defined as: Modest $(<2$ hours $)=$ ref.; Medium (2-3 hours or $>3$ hours maximum a few times/month); Extended ( $>3$ hours at least a few times/week). Computer work that puts a high demand on the eye-hand coordination was calculated as the sum of the number of hours using lay-out, graphics, CAD, design or similar software (CoordinationTime: Low $<0.25$ hours/day = ref; Medium 0,25-1.49; High $\geq 1.5$ ). Visual comfort was rated by 3 separate items (lighting conditions, glare from window or luminaries, reflexions from the computer screen) using a 9 step rating-scale: -4 (very, very bad) - 0 (indifferent) - +4 (very, very good). A Visual comfort index was calculated as an average value of these 3 items (VisualComfort, 3 levels). Comfort of screen position was rated using the same scale (ScreenComfort; 3 levels).

Subjects were also asked to register symptoms from different regions of the body (15 different: head, eyes, neck, right/left scapula, right/left shoulder/upper arm, right/left elbow/forearm, right/left wrist, right/left hand, upper back, low back) estimating the number of days during the preceding month with each specific symptom (e.g. eyes). During the follow up period 10 roughly monthly questionnaires asked about symptoms during the past month in a similar way as at baseline. Subjects reporting symptoms from the eyes during 0,1 or 2 days were considered as "symptom free" from the eyes, and those reporting symptoms $\geq 3$ days from the eyes were considered as having eye-symptoms. Subjects, who were symptom-free at baseline (or later if they had symptoms at baseline but recovered during the follow-up period and were symptom free during minimum one month) and reported symptoms in a later follow-up questionnaire, were considered as incident cases of eye-symptoms. The presence of eye symptoms at baseline was used as an explanatory variable (BaseSymptoms; No= ref; yes).

Subjects contributed with person-time under exposure (number of days) during the time-period between the date of the questionnaire when they were symptom free for the first time (at baseline or later) and the date of the questionnaire when they were considered as an incident eye case for the first time. Or the date of the last follow-up questionnaire if they remained symptom free.

Bivariate and multivariate associations between symptoms from the eyes and the explanatory variables were modeled as survival time models (Cox's proportional hazard model) with the response variable being time to case or last answered questionnaire. The explanatory variables from the baseline questionnaire were: ComputerTime, DeviceTime, CoordinationTime, ContinousTime, ScreenComfort, VisualComfort, Demands, Control, sex age, educational level, BMI, nicotine use and BaseSymptoms. A reduced multivariate model was arrived at using a stepwise selection process (entry $=0,25$ and stay $=0,20)$. For the analyses the TPHREG procedure (SAS 9.1.3) was used modeling the odds ratio for having versus for not having eye symptoms.

\section{Results}

During follow up time 329 of the 1246 subjects reported eye symptoms. The overall incidence rate of eye symptoms was 0.38 per person-year and 0.23 for subjects who were symptom free at base line and 1.06 for subjects with eye-symptoms at baseline. In the bivariate analyses significant associations were found between all explanatory variables, except BMI, and incident eye-symptoms. According to the reduced multivariate model significant associations were noted for BaseSymptoms (yes=higher risk), sex (women $=$ higher risk), ContinousTime (ex- 
tended=higher risk), VisualComfort (dose-response; worse $=$ higher risk) and nicotine use.

\section{Discussion}

The incidence of symptoms from the eyes was somewhat lower than the incidence of symptoms from the neck (0.67), shoulders $(0.41)$ or the arms/hands (0.47) in the same study group [2]. No other study has been found reporting incident eye symptoms among professional computer users. As was the case for neck and upper extremity disorders, women had a higher risk of getting eye symptoms than men. Visual discomfort was related to incident eye symptoms in a similar way as bad comfort of the computer work environment was to neck and upper extremity symptoms. Long-lasting computer work without breaks was also related to incident eye symptoms, in a similar way as was duration of mouse use to neck and upper extremity symptoms. Previous symptoms from the eyes increased the risk of new symptoms compared to those subjects without symptoms.

In conclusion, the incidence of eye problems among professional computer users is high and related to both individual and work-related factors.

\section{References}

[1] Karlqvist L, Wigaeus Tornqvist E, Hagberg M, Hagman M, Toomingas A. Self-reported working conditions of VDU operators and associations with musculoskeletal symptoms: a cross-sectional study focusing on gender differences. Int J Ind Ergon 2002;30:277-294.

[2] Wigaeus Tornqvist E, Hagberg M, Hagman M, Hansson Risberg E, Toomingas A. The influence of working conditions and individual factors on the incidence of neck and upper limb symptoms among professional computer users. Int Arch Occup Env Health 2009;82(6):689-702. 